\title{
The safety of echo contrast-enhanced ultrasound in high-intensity focused ultrasound ablation for abdominal wall endometriosis: a retrospective study
}

\author{
Sha Wang, Bo-Han Li, Jin-Juan Wang, Ying-Shu Guo, Jiu-Mei Cheng, Hong Ye, Chun-Yi Zang, \\ Ying Zhang, Hua Duan, Xiao-Ying Zhang \\ Department of Gynecologic Minimally Invasive Center, Beijing Obstetrics and Gynecology Hospital, Capital Medical University, Beijing, China \\ Correspondence to: Hua Duan; Xiao-Ying Zhang. Department of Gynecologic Minimally Invasive Center, Beijing Obstetrics and Gynecology Hospital, \\ Capital Medical University, No. 17, Qihe Lou Road, East District, Beijing 100006, China. Email: duanhuasci@163.com; xiaoyingzhang6731@aliyun.com.
}

Background: We aimed to investigate the efficacy and safety of echo contrast-enhanced ultrasound (CEUS) during high-intensity focused ultrasound (HIFU) ablation therapy for abdominal wall endometriosis (AWE). Methods: A total of 67 patients with AWE were treated with HIFU ablation, and their demographic characteristics were retrospectively analysed. Blood perfusion of the focal lesion was assessed before the operation, during ablation and after the operation with the use of an ultrasound contrast agent, and the effect of the ultrasound contrast agent on treatment was assessed over a 1-year follow-up period. The degree of symptom relief and adverse effects were evaluated after HIFU ablation.

Results: Eighty-two lesions were ablated in 67 patients. CEUS showed that all lesions were successfully ablated with HIFU. The shrinkage ratio of the lesions significantly increased over the follow-up period. Intermittent pain disappeared at 1 month after the operation, and the patients' pain scores significantly decreased at the 1 -year follow-up. The mean [ \pm standard deviation (SD)] lesion volume was $7.64 \pm 8.95 \mathrm{~cm}^{3}$ on B-mode ultrasound. The post-HIFU non-perfused volume was $18.34 \pm 24.08 \mathrm{~cm}^{3}$, and the rate of massive changes on greyscale imaging was $96.16 \% \pm 5.44 \%$ at 12 months. During the procedure, the main complications were a prickling sensation and tenderness in the treatment area and/or a transient "hot" sensation on the skin. After the procedure, there was no obvious discomfort except for pain. Two patients developed an approximately $1-\mathrm{cm}$ area of skin that exhibited a waxy appearance. Seven patients had haematuria. No severe complications were observed.

Conclusions: Ultrasound contrast agents are effective and safe for evaluating the effect of HIFU ablation on AWE, and this approach provides significant guidance and evaluation benefits for the use of HIFU treatment for AWE without obvious side effects.

Keywords: Echo contrast-enhanced ultrasound (echo CEUS); high-intensity focused ultrasound (HIFU); abdominal wall endometriosis (AWE); safety

Submitted Apr 28, 2020. Accepted for publication Nov 23, 2020.

doi: $10.21037 /$ qims-20-622

View this article at: http://dx.doi.org/10.21037/qims-20-622

\section{Introduction}

Abdominal wall endometriosis (AWE) is the most common endometriosis disease outside of the pelvic cavity. In recent years, with the increase in the caesarean section rate, the number of AWE cases has shown an obvious upward trend (1). AWE develops and invades the abdominal wall in different directions; it grows to the surface of the skin as a protrusion or into the abdominal cavity as adhesions to the surrounding organs, such as the intestine or bladder, and is one of the most common types of extra- 
pelvic endometriosis. Changes with periodic bleeding can also accompany the menstrual cycle. The most common symptom is a cyclic, painful mass or a palpable mass without pain. Surgical excision is still considered the most effective treatment procedure. However, the procedure causes further operative trauma and scars. High-intensity focused ultrasound (HIFU) ablation is a novel, non-invasive treatment for solid tumours that has been developed in recent years $(2,3)$.

Ultrasound-guided HIFU ablates AWE through significant ultrasound pressure-induced heat in a focused area. Each sonication procedure treats one spot at a time, line by line in a uniform pattern, conforming to the shape of the lesion being treated, and the non-perfusion volume is evaluated by echo contrast-enhanced ultrasound (CEUS) imaging. CEUS aims to assess tissue micro-vascularization $(2,4)$. This method utilizes convenient, B-mode imaging to provide outstanding images for diagnosis, HIFU positioning, and real-time monitoring by virtue of its excellent imaging properties, thus helping to evaluate and facilitate HIFU treatments $(5,6)$. Recently, CEUS has been proven safe during HIFU ablation of uterine fibroids and adenomyosis and is an effective method that has been evaluated and reinforced (7-9). Previous research has concluded that the gas bubbles applied as ultrasound contrast agents vibrate nonlinearly under tremendous acoustic pressures due to inertial cavitation, whereas heat emitting from high-frequency ultrasound with a large amount of energy may destroy adjacent tissue cells and is more likely metabolized by tissues (10). Cavitation has both advantages, as it may fortify HIFU thermal ablation, and disadvantages, as it may become uncontrolled and destroy the tissues further via ultrasonic wave scattering. However, it is not known whether the contrast agent plays an effective and safe role in HIFU-induced ablation of superficial tissue lesions.

Therefore, the present study aimed to explore the efficacy and safety of echo CEUS during HIFU ablation for AWE.

\section{Methods}

\section{Patients}

A total of 67 patients with AWE were studied in our hospital from March 2014 to September 2018, and their basic clinical data following HIFU were retrospectively collected. All patients signed consent forms preoperatively and before the use of echo contrast agents at the Capital Medical University, Beijing Obstetrics and Gynecology Hospital. The study was also approved by the hospital ethics board. The inclusion criteria for this study were as follows: (I) AWE diagnosed via ultrasound or magnetic resonance imaging (MRI), and HIFU physician onboard ultrasound location sound channel security (the distance of focus superficial to skin was $15 \mathrm{~mm}$, the distance of focus deep to abdominal was $15 \mathrm{~mm}$, and the lesion had a minimum diameter of $10 \mathrm{~mm}$ ); (II) clear ability to communicate with the physician and nurse; (III) no operation of the abdominal wall over the past 3 months, especially liposuction; and (IV) patients were without severe comorbidities, such as cardiopathy, hypertension, or diabetes, and could tolerate $2-3 \mathrm{~h}$ in a prone position $(2,11)$.

\section{CEUS and HIFU treatment}

SonoVue ${ }^{\circledR}$ (Bracco Suisse SA, Geneva, Switzerland), known as sulphur hexafluoride microbubbles, is an ultrasound contrast agent. First, the lyophilized powder was reconstituted with $5 \mathrm{~mL}$ of sodium chloride $0.9 \%$ w/v saline for injection according to the manufacturer's guidance. Prior to administration, the mixture was shaken for complete dissolution, and a bolus of $1.5-2.0 \mathrm{~mL}$ of the solution was administered, followed by $5 \mathrm{~mL}$ of saline immediately prior to HIFU treatment. Preprocedural CEUS was used to depict the perfusion and range of lesions (Figure $1 A, B$ ). As a routine, the contrast agent was administered twice: before treatment, the agent was injected to show the extent and blood supply of the lesion; then, as the treatment was concluding, the agent was administered to visualize the effects of the ablation. During treatment or prior to completion of the procedure, the solution was injected again to assess the HIFU thermal effect. If the CEUS results showed a blood supply in the lesion, the treatment would continue according to the skin condition of the treated area.

The preoperative preparation was the same as that in previous studies $(2,3)$. Routine diet control, such as with semi-liquids and clear enemas, and skin preparation, such as degreasing and degassing, were provided before treatment. The skin degreasing procedures were performed with alcohol, the degassing procedures were performed using a machine with negative pressure. The results mainly have an important role in the quality of ultrasound imaging. The patient is immersed in water, and there is no other interface between the water and the treated skin. The focused 

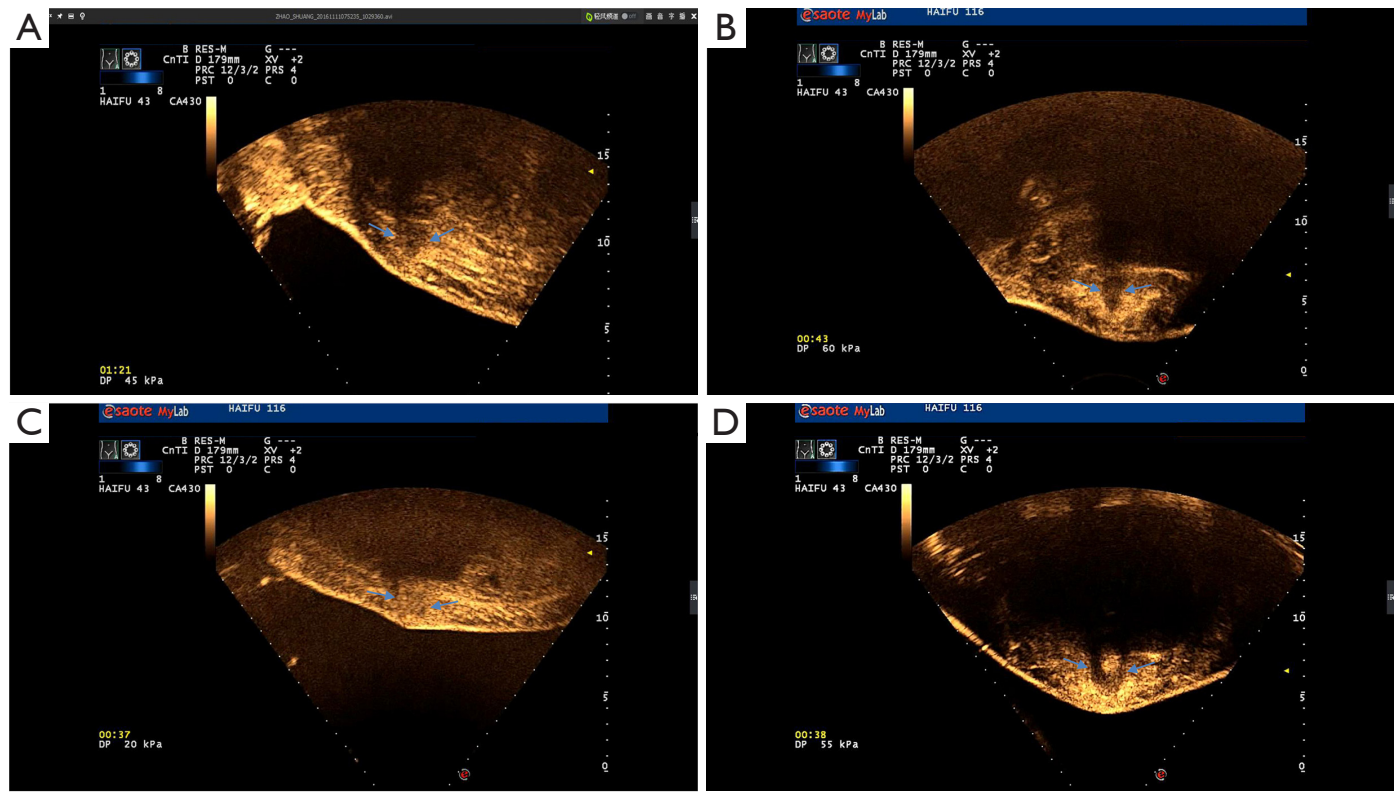

Figure 1 CEUS imaging of AWE foci before and after the HIFU procedure. (A) On the CEUS image, before HIFU treatment, an AWE mass shows hypoperfusion compared with the surrounding tissue; (B) on the CEUS image, before HIFU treatment, an AWE mass shows hyperperfusion compared with the surrounding tissue; (C) on the CEUS image immediately after HIFU treatment, the painful abdominal wall mass shows no perfusion; (D) on the CEUS image immediately after HIFU treatment, the painful abdominal wall mass shows no perfusion in the focal area. CEUS, contrast-enhanced ultrasound; AWE, abdominal wall endometriosis; HIFU, high-intensity focused ultrasound.

ultrasound therapy device (JC200, Chongqing, China) was configured with a focused frequency of $0.8 \mathrm{MHz}$, with an oval focus region with a long axis of $8 \mathrm{~mm}$ and a short axis of $3 \mathrm{~mm}$. During the procedure, the patients were kept in the prone position, the lower abdominal quadrant was soaked in cold water $\left(<10^{\circ} \mathrm{C}\right.$ as much as possible), and the bladder was constantly filled throughout the treatment process. Sedation and analgesia were maintained by an intravenous injection of fentanyl $(0.8-1 \mu \mathrm{g} / \mathrm{kg})$ and midazolam hydrochloride $(0.02-0.03 \mathrm{mg} / \mathrm{kg})$, which was repeated in $30-$ to $40-\mathrm{min}$ intervals according to the patient's pain tolerance at the focal area; simultaneously, the patient was monitored for burns to the skin during the treatment process.

HIFU was initiated 10 min after the contrast agent injection. First, the treatment algorithm was arranged according to the longitudinal range (from the left edge to the right edge) of the focal lesion in sequential layers of $3-5 \mathrm{~mm}$. Second, the focal spot was maintained on the lesion, with a $5-10 \mathrm{~mm}$ safe distance from the focus to the skin and/or abdominal cavity; a mean power of $150 \mathrm{~W}$ was applied for $1 \mathrm{~s}$ with a 3 -s interval at each point, then stopped or moved to another site after treatment was administered for approximately 5-8 s or upon appearance of the "popcorn" sign. In this manner, point-to-point HIFU treatment was performed with an ablation power of 100-300 W, which was adjusted according to the patient's response and/or the level of change in the lesion being treated under real-time ultrasound monitoring and evaluation. If micro-vascular perfusion occurred in the treated lesion, the thermal treatment was continued in the same session according to the skin's response to heat in the treated area and the development of any induration of the skin or subcutaneous tissue. After HIFU, CEUS of the ablated region showed a dot representing the non-perfusion region (Figure $1 C, D$ ). After HIFU, the patients relaxed in the prone position, and the treated lesions were immersed in cool water for $30 \mathrm{~min}$. The bladder was infused twice with cool water, which was retained for approximately 20-30 min.

\section{Follow-up}

Subsequently, the patients were followed up from 1 to 12 months, and any adverse sides or complications that met 
the standard of the International Radiological Association were recorded.

\section{Statistical analysis}

All data are expressed as the mean \pm standard deviation (SD). Statistical analysis was performed using SPSS software (version 21.0, IBM Corp., Armonk, NY, USA). The normality of the data was assessed using the Shapiro-Wilks test, with $\mathrm{P}>0.05$ indicating a normal distribution. Student's $t$-tests were performed for normally distributed data to compare pairs of samples. Wilcoxon matched-pairs tests were performed for non-normally distributed data. $\mathrm{P}<0.05$ and $\mathrm{P}<0.01$ indicated a significant difference and highly significant difference, respectively.

\section{Results}

\section{Demographic characteristics of patients with AWE}

The demographic characteristics are shown in detail in Table 1. As shown in Figure 2, 134 patients with pain when the mass was palpated participated in localized treatment with ultrasound. Among those patients, 33 were excluded: 10 did not meet the inclusion criteria for having an abdominal wall thickness less than $10 \mathrm{~mm}$ or had obstacles to treatment such as adhesions or abdominal tissue, and 23 declined to participate. Among the 101 participants, 34 subjects withdrew before the trial ended. Three did not receive HIFU due to inpatient fees or concerns about AWE ablation remaining in the abdominal wall. Fifteen patients did not have obvious symptoms, such as periodic abdominal pain or a lesion diameter that was smaller than $10 \mathrm{~mm}$, and 16 were excluded from HIFU treatment after observation because the nature of the lesion was not clear or due to concerns regarding complications. Finally, 67 patients with AWE were included in the study. All participants completed a follow-up examination at 12 months.

\section{Baseline imaging features of AWE}

As shown in Table 2, the texture of $100 \%(82 / 82)$ of the lesions was hard, and the degree of activity was high in $11 \%(9 / 82)$ and low in $89 \%(73 / 82)$ of the included lesions. A total of $82 \%(67 / 82)$ of the lesions exhibited adhesions to the surrounding rectal tissue, while $18 \%(15 / 82)$ were located in the subcutaneous fat layer. There was no difference between the lesions located to the left of the scar $(38 \%)$ and those located to the right (39\%). A total of $73 / 82$ of the lesions had low activity. Most of the lesions $(63 / 82)$ had invaded the rectus, and 4/82 were in the fascia. Although $54 / 82$ of the patients had single lesions, 7/82 patients had two lesions, and 6/82 had three lesions. A total of $59.76 \%$ of the lesions did not protrude and were not prominent on the surface of the skin. A total of $76 / 82$ of the lesions had a visual analogue scale (VAS) score of 3.0 \pm 1.4 .

\section{The results of patients with AWE after HIFU treatment}

As shown in Table 3, after HIFU treatment, the median non-perfused lesion volume was $18.3 \pm 24.1 \mathrm{~cm}^{3}$, the rate of massive greyscale changes was $90 \%$, the median total treatment time was 39.5 [14-113] min, the median total sonication time was $245[71-1,200] \mathrm{s}$, and the total sonication volume was $3.6(1.35-12.83) \mathrm{cm}^{3}$. The median sonication time for $1 \mathrm{~cm}^{3}$ was $69.3(34.7-$ 137.34) s, while the median total energy was 36,575 $[10,650-183,000] \mathrm{J}$, with a median sonication intensity of $431(103.45-977.14) \mathrm{s} / \mathrm{h}$, and the median energy efficiency factor (EEF) was 10,042.9 $(4,935-24,888.89) \mathrm{J} / \mathrm{cm}^{3}$. The intra-procedural median VAS score was 6 [0-10], and the post-procedural median VAS score was 0 [0-7]. There was a significant difference between intra- and post-procedural VAS scores $(\mathrm{P}<0.05)$.

\section{The comparison of pre-and post-procedure two-dimensional (2D) and CEUS evaluations of patients with AWE}

As shown in Table 4, a difference existed between the 2D and CEUS evaluations of patients before the procedure, as well as between the CEUS measurements acquired before and after HIFU ablation of AWE.

\section{Intra-procedural and post-procedural side effects}

As shown in Table 5, no adverse events correlated with CEUS during or after HIFU ablation for AWE. The adverse effects or complications following HIFU treatment are shown in Table 5. A total of 12 patients experienced Society of Interventional Radiology (SIR) class A skin thermalgia, which appeared once or twice for only $1-2 \mathrm{~s}$ during thermal ablation, and 67 patients had tenderness in the treatment area classified as SIR class B that occurred during treatment and did not disappear until approximately $2 \mathrm{~h}$ after treatment. There were two patients who developed 
Table 1 Demographic characteristics of patients with AWE

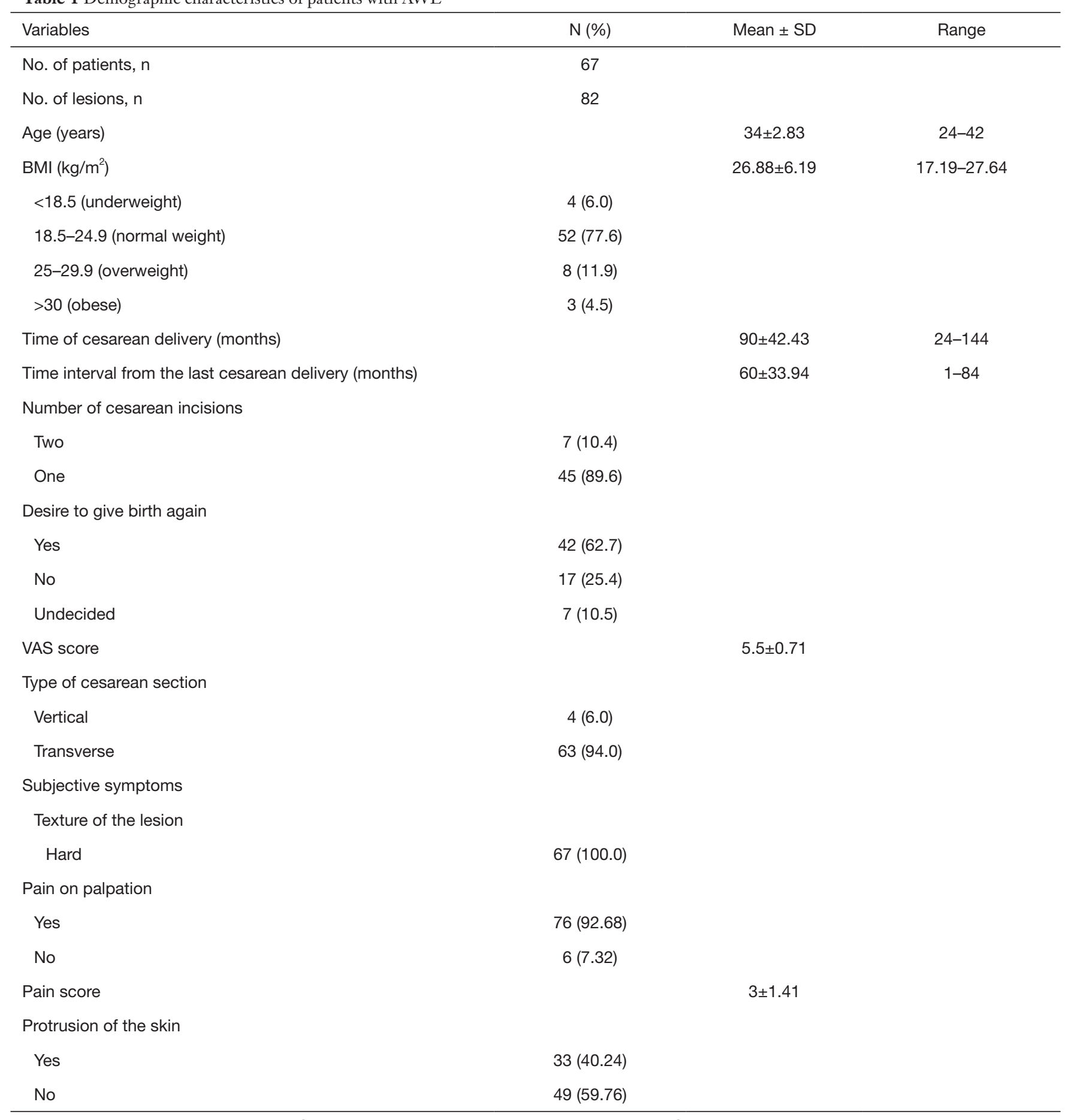

AWE, abdominal wall endometriosis; SD, standard deviation; BMI, body mass index; VAS, visual analogue scale. 


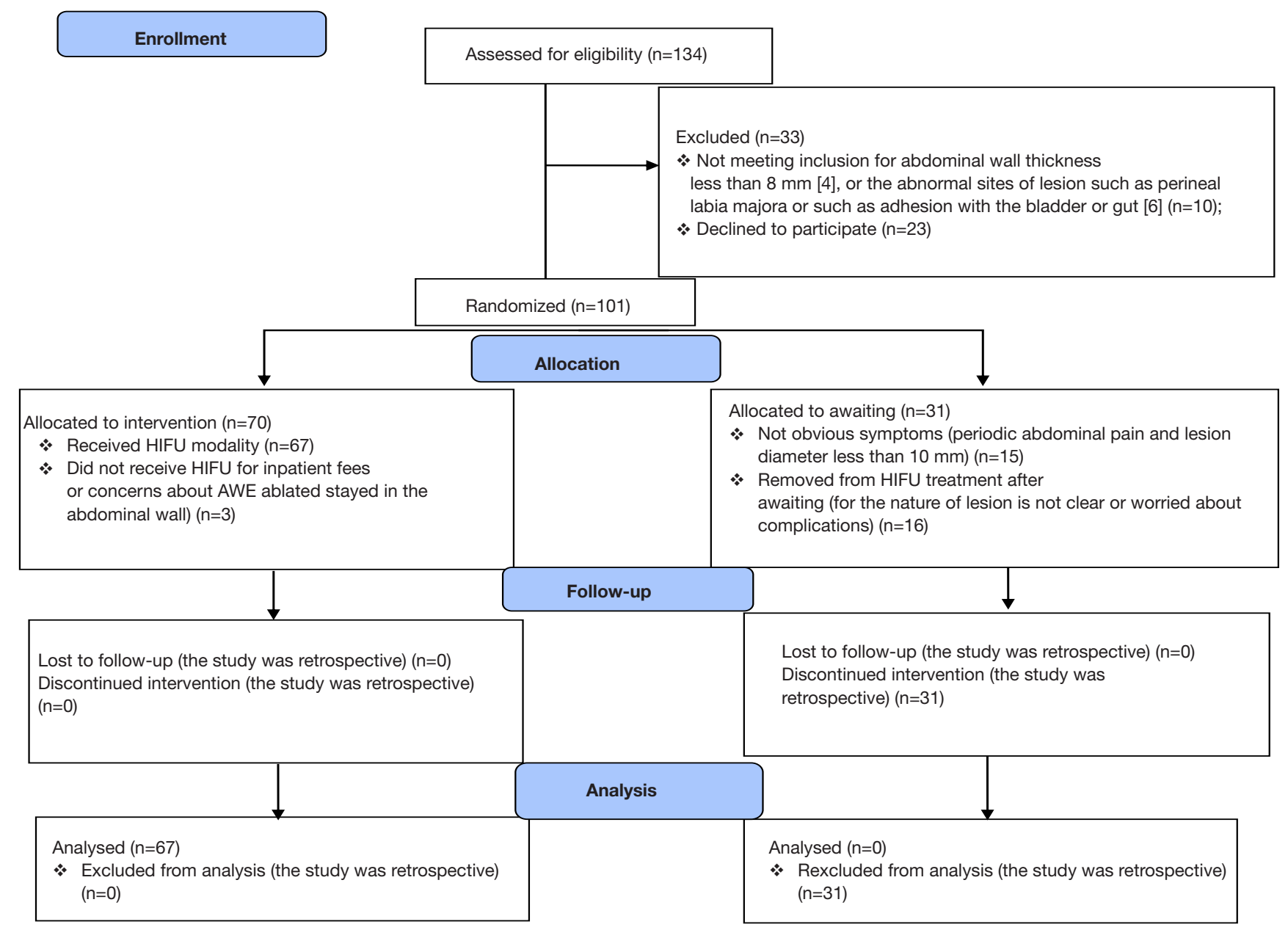

Figure 2 CONSORT flow diagram of CEUS in HIFU. CEUS, contrast-enhanced ultrasound; HIFU, high-intensity focused ultrasound; AWE, abdominal wall endometriosis.

an orange peel-like appearance on the skin with a waxy centre approximately $1.0 \mathrm{~cm}$ in diameter; one patient was cured without any treatment after 1 month, and the other was cured after 5 months (Figure $3 A, B$ ). Seven patients had haematuria; three cases occurred during treatment, and four cases occurred after treatment. These patients recovered without any medication.

\section{Follow-up of patients with AWE after HIFU treatment}

At 12 months of follow-up, no patient had periodic pain. Their VAS scores significantly decreased from $2.00 \pm 0.00$ at 1 month and $1.00 \pm 1.41$ at 6 months to $0.5 \pm 0.71$ at 12 months (Table 6). In addition, the rate of decrease in volume increased from $25.34 \% \pm 18.83 \%$ at 1 month to
$81.89 \% \pm 15.69 \%$ at 6 months (Figure $1 A$ ) $96.16 \% \pm 5.44 \%$ at 12 months. Two patients had skin burn injuries; one recovered 15 days post-treatment with no intervention, and the other recovered after 5 months with topical medication.

\section{Discussion}

In recent years, HIFU has been shown to be a feasible and effective non-invasive modality to ablate AWE. HIFUrelated side effects or complications are mild or rare during and/or after HIFU, especially for thermal ablation for AWE. Many studies have shown that HIFU ablation is safe for AWE and other uterine diseases (12); our study obtained similar results $(13,14)$. In our study, we conducted a 1 -year follow-up. The results of the follow-up showed that HIFU 
Table 2 Results of imaging features of AWE before HIFU procedure

\begin{tabular}{|c|c|c|}
\hline Variables & $N(\%)$ & Mean \pm SD \\
\hline \multicolumn{3}{|l|}{ Diagnostic modality } \\
\hline Ultrasonography & $67(100)$ & \\
\hline MRI & $8(11.94)$ & \\
\hline \multicolumn{3}{|l|}{ Nodule location } \\
\hline To the left of the scar & $31(37.81)$ & \\
\hline At the middle of the scar & $19(23.17)$ & \\
\hline To the right of the scar & $32(39.02)$ & \\
\hline \multicolumn{3}{|l|}{ Texture of nodules } \\
\hline Homogeneous hypo-echo & $76(92.68)$ & \\
\hline Heterogeneous hypo-echo & $4(4.88)$ & \\
\hline Hyper-echo & $2(2.44)$ & \\
\hline \multicolumn{3}{|l|}{ Degree of activity } \\
\hline High & $9(10.98)$ & \\
\hline Low & $73(89.02)$ & \\
\hline \multicolumn{3}{|l|}{ Adhesions to the surrounding tissue } \\
\hline Subcutaneous fat layer and rectus & $63(76.83)$ & \\
\hline Subcutaneous fat layer & $15(18.29)$ & \\
\hline Fascial & $4(4.89)$ & \\
\hline Thickness of the rectus, $\mathrm{mm}^{*}$ & & $11.3 \pm 6.65$ \\
\hline Thickness of the fat, $\mathrm{mm}$ & & $23.3 \pm 6.65$ \\
\hline \multicolumn{3}{|l|}{ Number of nodules } \\
\hline Single nodule & $54(65.85)$ & \\
\hline Two nodules & 7 (8.54) & \\
\hline Three nodules & $6(7.32)$ & \\
\hline
\end{tabular}

*, $\mathrm{P}<0.01$, Student's $t$-tests. AWE, abdominal wall endometriosis; HIFU, high-intensity focused ultrasound; SD, standard deviation; $\mathrm{MRI}$, magnetic resonance imaging.

treatment under the echo CEUS could effectively alleviate the patient's pain while significantly reducing the size of the lesion. No adverse events correlated with the usage of CEUS recorded during or after the operation or at the 1-year follow-up postoperatively.

According to previous studies, there are no clear inclusion and exclusion criteria. As long as there is the acoustic channel in the lesion, it can be treated. Because the size of the oval focus region is a long axis of $8 \mathrm{~mm}$, the safe treatment path is at least the distance of focus superficial
Table 3 Results of patients with AWE after HIFU treatment

\begin{tabular}{lll}
\hline Variables & Mean $\pm \mathrm{SD}$ & $\mathrm{N}(\%)$ \\
\hline Lesion volume, $\mathrm{cm}^{3}$ & $7.64 \pm 8.95$ \\
Non-perfused volume, $\mathrm{cm}^{3}$ & $18.34 \pm 24.08$ \\
Rate of non-perfused volume, \% & $1.77 \pm 1.08$ \\
Average power, J & $133.50 \pm 23.34$ \\
Total treatment time, min & $64 \pm 36.77$ \\
Total sonication time, s & $371 \pm 29.70$ \\
Total sonication volume, $\mathrm{cm}^{3}$ & $5.70 \pm 1.27$ \\
Sonication time for $1 \mathrm{~cm}^{3}, \mathrm{~s} / \mathrm{cm}^{3}$ & $75.76 \pm 32.18$ \\
Sonication intensity, $\mathrm{s} / \mathrm{h}$ & $426.14 \pm 272.67$ \\
Total energy, J & $49,900 \pm 12,586.5$ \\
EEF, J/cm & \\
CEUS evaluation of lesion & $9,231.06 \pm 4,269.43$ & \\
incomplete & \\
Intraprocedural VAS & \\
Postprocedural VAS & $5.50 \pm 0.71$ \\
\hline
\end{tabular}

*, $\mathrm{P}<0.01$, Student's $t$-tests. AWE, abdominal wall endometriosis; HIFU, high-intensity focused ultrasound; SD, standard deviation; EEF, energy efficiency factor; CEUS, contrast-enhanced ultrasound; VAS, visual analogue scale.

to skin was $15 \mathrm{~mm}$, and the distance of focus vdeep to abdominal cavity was $10 \mathrm{~mm}$. Moreover, according to the study of Song et al. (15), the consistency between the clinical diagnosis of AWE and the postoperative pathology is only approximately $50 \%$. Due to the lack of postoperative pathological diagnosis in HIFU treatment, the periodic abdominal wall pain and other symptoms related to endometriosis could be confirmed to assist in the clinical diagnosis of AWE. Therefore, we suggest adding regular abdominal wall pain as an indication for HIFU treatment.

SonoVue ${ }^{\circledR}$ is widely used clinically to diagnose or evaluate the nature of a lesion or therapeutic effects. The majority of studies have used CEUS to evaluate HIFU treatment focused on liver and prostate diseases (16), and there have been few reports in obstetrics and gynaecology. According to previous research, CEUS is the ideal imaging examination to monitor HIFU treatment. Microbubbles may not affect kidney or thyroid function; thus, CEUS can be repeated several times without impairing renal function due to the use of a contrast agent or radiation exposure to the patient. Compared to MRI or computed tomography 
Table 4 Comparison of 2D and CEUS evaluation of patients with AWE pre-procedure and post-procedure $(\mathrm{mean} \pm \mathrm{SD})$

\begin{tabular}{|c|c|c|c|}
\hline Variable & $\frac{2 \mathrm{D}}{\text { Before treatment }}$ & \multicolumn{2}{|c|}{ CEUS } \\
\hline Lesion volume, $\mathrm{cm}^{3}$ & $7.64 \pm 8.95$ & $10.32 \pm 12.75$ & $18.34 \pm 24.08$ \\
\hline
\end{tabular}

Table 5 Side effects or complications of patients with AWE during and/or after HIFU treatment $(\mathrm{n}=67)$

\begin{tabular}{lll}
\hline SIR class & Complications & No. \\
\hline A & Skin thermalgia & 12 \\
B & Pain in the treatment area & 67 \\
& Skin blistering & 1 \\
N/A & haematuria & 7 \\
N/A & Skin damage & 1 \\
\hline
\end{tabular}

AWE, abdominal wall endometriosis; HIFU, high-intensity focused ultrasound; SIR, Society of Interventional Radiology.

(CT), CEUS is simple to perform, widely available and inexpensive (16). Compared with simple ultrasound, CEUS can more clearly show the range of lesions by comparing development and distribution over time (17). Compared with 2D ultrasound, CEUS showed more obvious AWE lesions in this trial. Regarding safety, Cheng et al. (18) conducted a study evaluating CEUS for HIFU treatment in benign gynaecological diseases. The results suggested that there was no significant increase in adverse events after applying SonoVue, and CEUS can reduce the abdominal pain caused by an inaccurate ablation range. In general, SonoVue ${ }^{\circledR}$ is safe and effective for evaluating HIFU ablation in uterine disease. However, there is a difference between the use of HIFU therapy for AWE and for uterine diseases. During HIFU ablation on superficial tissue, attention was paid to preventing the cavitation effect from damaging the normal tissue around the lesion, especially the skin in front of the acoustic path, or adjacent organs such as the bladder or intestine behind the acoustic path. There are obvious features of endometriotic lesions of the uterus and the abdominal wall that react differently to HIFU thermal deposition. Generally, cavitation damage to the surrounding tissue can be prevented during HIFU ablation for AWE. As a routine procedure, thermal treatment began $10 \mathrm{~min}$ after contrast administration so that SonoVue ${ }^{\circledR}$ was washed out from the blood circulatory system to alleviate the effects of gas cavitation.

Moreover, during ultrasound-guided HIFU ablation for AWE, the appearance of the "popcorn effect" was monitored in all study patients. This sign is an indication of over-treatment of the target region (19) and can be controlled to prevent diffusion, propagation, and thermal destruction of the surface of the skin and/or organs under the abdominal cavity.

When treatment is almost completed, CEUS was performed in the operating room to evaluate the HIFU ablation effect and to determine whether residual perfusion existed in the targeted area. On B-mode sonographic imaging, greyscale patterns in the focused lesion might not show obvious changes. Thus, the results of thermal ablation cannot be judged only by 2D ultrasonic imaging. The nonperfusion value (NPV) is typically assessed as the index in non-invasive treatment modalities, and CEUS is considered to be as accurate as other contrast-enhanced imaging modalities $(17,20)$. Based on the NPV, if micro-diffusion occurs in the targeted area, re-ablation cannot be performed until 10 min after the injection of a contrast agent in the region of the residual lesion. This method is common practice in our institution and led to the identification of incompletely ablated lesions in 4/67 (5.97\%) patients during the HIFU process. Among them, $100 \%$ of the incompletely ablated lesions were successfully re-ablated.

Therefore, the use of an ultrasound contrast agent to preoperatively evaluate the focal area could help determine the blood perfusion of the area and to highlight the range of the lesions. Indeed, there was an obvious significant difference in lesion volume between 2D and CEUS measurements acquired before the procedure. The use of a contrast agent could distinguish the lesion from surrounding normal tissue, making it easier to define the ablation area of the lesions. Otherwise, because of the influence of greyscale features, it is difficult for CUES to assess the effects of thermal ablation for such lesions. The reasons for 

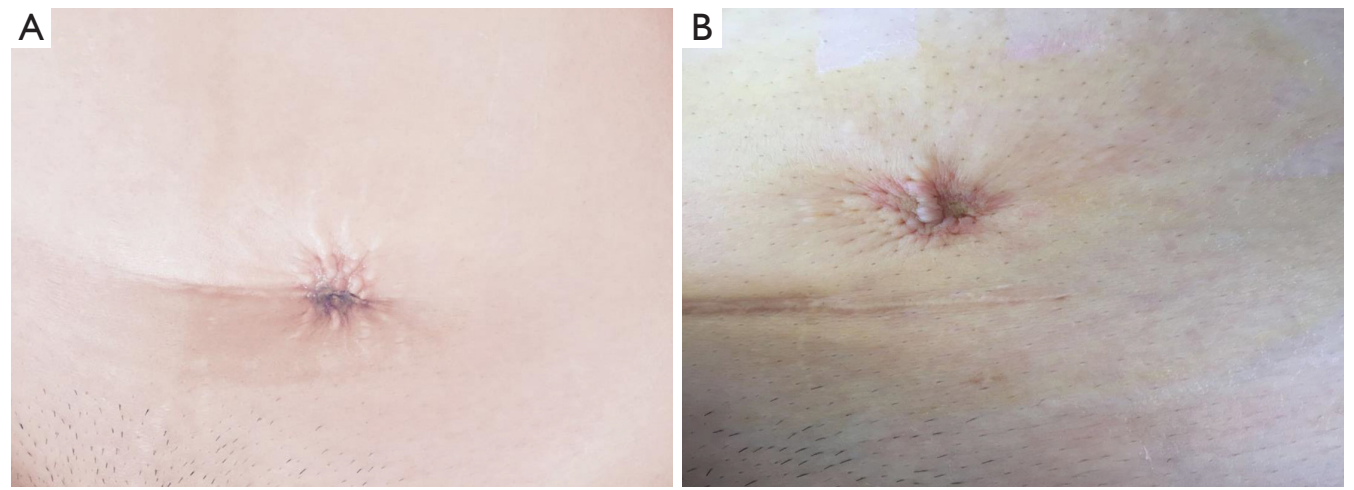

Figure 3 Skin images of AWE foci after the HIFU procedure. (A) Skin has an orange peel-like appearance with a waxy centre. The diameter was $>1.0 \mathrm{~cm}$ on the second day after treatment; (B) skin has an orange peel-like appearance with a waxy centre. The diameter was $<1.0 \mathrm{~cm}$ on the second day after treatment. AWE, abdominal wall endometriosis; HIFU, high-intensity focused ultrasound.

Table 6 Follow-up of patients with AWE after treatment with HIFU ablation

\begin{tabular}{lcccc}
\hline Variables & Before treatment & 1 month $(\mathrm{n}=67)$ & 6 months $(\mathrm{n}=61)$ & 12 months $(\mathrm{n}=58)$ \\
\hline VAS & $5.5 \pm 0.71$ & $2.00 \pm 0.00^{*}$ & $1.00 \pm 1.41^{*}$ & $0.5 \pm 0.71^{*}$ \\
Rate of volume decrease (\%) & & $16.70 \pm 6.60$ & $81.89 \pm 15.69^{*}$ & $96.16 \pm 5.44^{*}$ \\
\hline
\end{tabular}

*, $P<0.01$, Student's $t$-tests. AWE, abdominal wall endometriosis; HIFU, high-intensity focused ultrasound; VAS, visual analogue scale.

the significant differences in CEUS measurements before and after ablation include oedema in the peripheral tissue due to thermal effects, etc. During HIFU ablation of the superficial tissue, attention is paid to controlling not only the intensity of sonication but also the sonication time per $\mathrm{cm}^{3}$ to prevent skin burning. There is a major difference in applying CEUS during HIFU treatment to the superficial tissue and the uterus.

In this study, only two patients sustained skin burn injuries. In-depth analysis of the causes showed that with large lesions, too much energy was focused on the area within too short a time, or the patient moved unconsciously when thermal ablation began. Moreover, when a "popcorn" effect appeared, the treatment had to be adjusted slowly to prevent propagation of the effect. In addition, intraor post-procedural haematuria occurred in seven patients due to the ultrasound dispersion of gas in the bladder. The causes of gas in the bladder are as follows: the intravesical fusion of saline is not completely degassed, and/or when the nurse inserted the catheter into the bladder before treatment, a small amount of urine was not retained in the bladder to evacuate the gas in the catheter. These details, as described above, can be addressed before therapy to avoid gas in the bladder.
If intra-procedural haematuria occurred, thermal ablation was not started until the gas in the bladder had disappeared; after treatment, the bladder was bathed in cool saline for 30 min until the urine was clear. Regardless of when the saline bath occurred, we needed to infuse the bladder with iced saline infusion to clear the urine; moreover, after treatment, the patients were required to drink more water and urinate more frequently to prevent urinary tract infections, and oral anti-inflammatory drugs were given to prevent urinary tract infections when necessary. None of the patients developed urinary tract complications during follow-up. In the HIFU process, no adverse events correlating with the usage of CEUS were recorded during or after the operation or at the 1-year follow-up postoperatively.

During the procedure, skin thermalgia or pain in the treatment area occurred. First, the skin in the treatment area was touched to determine whether it was soft. The softness of the skin was categorized as follows: soft, like lips; medium, like the nose tip; and hard, like the forehead. This evaluation should be routinely performed every 10-20 min during treatment. Then, as long as the skin is soft, the treatment should continue; pain was considered a normal response to the treatment zone. Immediately after treatment, patients experienced swelling and pain 
in the focal region that typically resolved without any therapy after approximately $2 \mathrm{~h}$. Thereafter, patients could return to normal activity. Periodic pain disappeared in all patients with AWE; moreover, the volumes of the lesions were obviously reduced 1 month after HIFU ablation. In contrast to surgery, HIFU ablation for AWE is confined to the abdominal wall, and the lesions are slowly absorbed and consistently decrease in size $(21,22)$. However, ablation is unable to determine the pathological nature of the lesions. These are the disadvantages and advantages of ablation for abdominal wall lesions, especially for larger lesions; however, ablation can maintain abdominal wall integrity and treat multiple lesions while avoiding multiple incisions. Furthermore, even if the ablated mass recurs, it is convenient and feasible for HIFU therapy to be repeated as a non-invasive treatment. As recent studies have shown, the superior imaging features of CEUS can detect and evaluate the effect of thermal ablation in real-time during HIFU ablation without side effects (2,3). HIFU has also been shown to be practical and safe for superficial tissues $(2,3)$. In this study, CEUS was shown to be an effective, real-time, non-invasive modality that allowed for multiple scans in the longitudinal and transverse planes. Thus, this method represents one of the most promising tools to tailor and monitor the HIFU process. The main goal of the study focused on the safety and efficacy of the echo contrast ultrasound in HIFU ablation for AWE, not on the efficacy and safety of HIFU ablation for AWE. According to current research, the contrast agent is metabolized $10 \mathrm{~min}$ after injection. According to the research of $\mathrm{Hu}$ et al. (23), the known adverse reactions of CEUS intraoperative application included anaphylactic shock, skin allergies, nausea or vomiting, dizziness or headache, numbness, chest distress, back pain, and local reactions of the injection site. These reactions mostly appear within $24 \mathrm{~h}$ after surgery. No long-term adverse reactions have been found. In the study of Chen et al. (9), after applying CEUS intraoperatively for patients with uterine fibroids and a 1-week follow-up, the authors found no related longterm adverse reactions. Therefore, combined with our research results, we preliminarily believe that CEUS is safe for HIFU treatment of AWE. However, there were several limitations in this study, including the small sample size and the retrospective nature of the study, as well as the lack of a quantitative analysis of CEUS and a contrast study.

\section{Conclusions}

The use of ultrasound contrast agents is an effective and safe method for evaluating the effect of HIFU ablation of AWE. Furthermore, in the clinical setting, CEUS showed significant guidance and evaluation benefits for the HIFU treatment of abdominal wall adenomyosis without any obvious short- or long-term side effects.

\section{Acknowledgments}

We thank our medical team for their support and assistance. Funding: This study was funded by a Natural Science Foundation of China grant (grant number 81571412) and the Beijing Municipal Administration of Hospital Clinical Medicine, Development of Special Funding Support (grant number ZYLX201406).

\section{Footnote}

Conflicts of Interest: All authors have completed the ICMJE uniform disclosure form (available at http://dx.doi. org/10.21037/qims-20-622). The authors have no conflicts of interest to declare.

Ethics Statement: Our study protocol has been approved by the local ethics committee and is in accordance with the Helsinki declaration. Written informed consent was obtained from the patient for publication of this study and any accompanying images.

Open Access Statement: This is an Open Access article distributed in accordance with the Creative Commons Attribution-NonCommercial-NoDerivs 4.0 International License (CC BY-NC-ND 4.0), which permits the noncommercial replication and distribution of the article with the strict proviso that no changes or edits are made and the original work is properly cited (including links to both the formal publication through the relevant DOI and the license). See: https://creativecommons.org/licenses/by-nc-nd/4.0/.

\section{References}

1. Zhao L, Deng Y, Wei Q, Chen J, Zhao C. Comparison of ultrasound-guided high-intensity focused ultrasound ablation and surgery for abdominal wall endometriosis. Int 
J Hyperthermia 2018;35:528-33.

2. Zhu X, Chen L, Deng X, Xiao S, Ye M, Xue M. A comparison between high-intensity focused ultrasound and surgical treatment for the management of abdominal wall endometriosis. BJOG 2017;124 Suppl 3:53-8.

3. Xiao-Ying Z, Hua D, Jin-Juan W, Ying-Shu G, JiuMei C, Hong Y, Chun-Yi Z. Clinical analysis of highintensity focussed ultrasound ablation for abdominal wall endometriosis: a 4-year experience at a specialty gynecological institution. Int J Hyperthermia. 2019;36:87-94.

4. Dong Y, Braden B, Klinger C, Ripolles T, Dietrich CF. Ultrasound findings in extragenital endometriosis. J Ultrason 2018;18:247-54.

5. Peng S, Xiong Y, Li K, He M, Deng Y, Chen L, Zou M, Chen W, Wang Z, He J, Zhang L. Clinical utility of a microbubble-enhancing contrast ("SonoVue") in treatment of uterine fibroids with high intensity focused ultrasound: a retrospective study. Eur J Radiol 2012;81:3832-8.

6. Peng S, Hu L, Chen W, Chen J, Yang C, Wang X, Zhang $\mathrm{R}$, Wang Z, Zhang L. Intraprocedure contrast enhanced ultrasound: the value in assessing the effect of ultrasoundguided high intensity focused ultrasound ablation for uterine fibroids. Ultrasonics 2015;58:123-8.

7. Isern J, Pessarrodona A, Rodriguez J, Vallejo E, Gimenez N, Cassadó J, De Marco JA, Pedrerol A. Using microbubble sonographic contrast agent to enhance the effect of high intensity focused ultrasound for the treatment of uterine fibroids. Ultrason Sonochem. 2015;27:688-93.

8. Cheng C, Xiao Z, Huang G, Zhang L, Bai J. Enhancing ablation effects of a microbubble contrast agent on highintensity focused ultrasound: an experimental and clinical study. BJOG 2017;124 Suppl 3:78-86.

9. Chen Y, Jiang J, Zeng Y, Tian X, Zhang M, Wu H, Zhou H. Effects of a microbubble ultrasound contrast agent on high-intensity focused ultrasound for uterine fibroids: a randomised controlled trial. Int J Hyperthermia 2018;34:1311-5.

10. Coussios CC, Farny CH, Haar GT, Roy RA. Role of acoustic cavitation in the delivery and monitoring of cancer treatment by high-intensity focused ultrasound (HIFU). Int J Hyperthermia 2007;23:105-20.

11. Zhang X, Duan H. Effect of high-intensity focused ultrasound ablation on endometriosis of the abdominal wall. Int J Clin Exp Pathol 2018;11:2118-24.

12. Zhang X, Duan H. One-time high-intensity focused ultrasound ablation of abdominal wall endometriosis with concurrent uterine fibroids or adenomyosis: two cases and literature review. Quant Imaging Med Surg 2020;10:511-7.

13. Liu X, Wang W, Wang Y, Wang Y, Li Q, Tang J. Clinical predictors of long-term success in ultrasound-guided high-intensity focused ultrasound ablation treatment for adenomyosis: a retrospective study. Medicine (Baltimore) 2016;95:e2443.

14. Wang W, Wang Y, Tang J. Safety and efficacy of high intensity focused ultrasound ablation therapy for adenomyosis. Acad Radiol 2009;16:1416-23.

15. Song H, Lee S, Kim MJ, Shin JE, Lee DW, Lee HN. Abdominal wall mass suspected of endometriosis: clinical and pathologic features. Obstet Gynecol Sci 2020;63:357-62.

16. Apfelbeck M, Clevert DA, Ricke J, Stief C, Schlenker B. Contrast enhanced ultrasound (CEUS) with MRI image fusion for monitoring focal therapy of prostate cancer with high intensity focused ultrasound (HIFU) 1. Clin Hemorheol Microcirc 2018;69:93-100.

17. Sidhu PS, Cantisani V, Dietrich CF, Gilja OH, Saftoiu A, Bartels E, Bertolotto M, Calliada F, Clevert DA, Cosgrove D, Deganello A, D'Onofrio M, Drudi FM, Freeman S, Harvey C, Jenssen C, Jung EM, Klauser AS, Lassau N, Meloni MF, Leen E, Nicolau C, Nolsoe C, Piscaglia F, Prada F, Prosch H, Radzina M, Savelli L, Weskott HP, Wijkstra H. The EFSUMB guidelines and recommendations for the clinical practice of contrast-enhanced ultrasound (CEUS) in non-Hepatic applications: update 2017 (short version). Ultraschall Med 2018;39:154-80.

18. Cheng CQ, Zhang RT, Xiong Y, Chen L, Wang J, Huang GH, Li KQ, Zhang L, Bai J. Contrast-enhanced ultrasound for evaluation of high-intensity focused ultrasound treatment of benign uterine diseases: retrospective analysis of contrast safety. Medicine (Baltimore) 2015;94:e729.

19. Farny CH, Holt RG, Roy RA. Temporal and spatial detection of HIFU-induced inertial and hot-vapor cavitation with a diagnostic ultrasound system. Ultrasound Med Biol 2009;35:603-15.

20. Mechl M, Šprláková-Puková A, Foukal J, Šmajerová M, Bohatá Š, Válek V. Contrast-enhanced ultrasonography. Cas Lek Cesk 2018;157:175-80.

21. Nguyen MD. Magnetic resonance imaging-guided highintensity focused ultrasound ablation for endometriosis of the abdominal wall. Gynecol Minim Invasive Ther 
2020;9:45-6.

22. Wang Y, Wang W, Wang L, Wang J, Tang J. Ultrasoundguided high-intensity focused ultrasound treatment for abdominal wall endometriosis: preliminary results. Eur J
Radiol 2011;79:56-9.

23. Hu C, Feng Y, Huang P, Jin J. Adverse reactions after the use of SonoVue contrast agent: Characteristics and nursing care experience. Medicine (Baltimore) 2019;98:e17745.

Cite this article as: Wang S, Li BH, Wang JJ, Guo YS, Cheng JM, Ye H, Zang CY, Zhang Y, Duan H, Zhang XY. The safety of echo contrast-enhanced ultrasound in high-intensity focused ultrasound ablation for abdominal wall endometriosis: a retrospective study. Quant Imaging Med Surg 2021;11(5):17511762. doi: 10.21037/qims-20-622 